\title{
COMPORTAMENTO INFORMACIONAL DOS PROFISSIONAIS DA SAÚDE NO HOSPITAL DE REABILITAÇÃO DE ANOMALIAS CRANIOFACIAIS DA UNIVERSIDADE DE SÃO PAULO - HRAC - USP
}

\begin{abstract}
Resumo: Os estudos de comportamento informacional visam descrever o comportamento humano relacionado às fontes e canais de informação, descrevendo os processos de busca, recuperação, acesso e uso da informação. As contribuições destes estudos, quando seguem a abordagem de domínio, podem ser aplicadas em produtos e serviços informacionais adequados a diferentes grupos de usuários de estudados. Neste estudo, buscou-se descrever as necessidades informacionais de membros de uma equipe multidisciplinar de profissionais da área da saúde, em um hospital especializado em reabilitação de anomalias craniofaciais. A coleta de dados foi realizada por meio de aplicação de questionário aos profissionais da equipe de atendimento aos pacientes com fissura labiopalatina, incluindo as áreas de medicina, odontologia, enfermagem, fonoaudiologia, serviço social e psicologia, num total de 56 participantes. Os resultados indicaram uma preferência dos participantes por fontes de informação informais (consultas a colegas e/ou à equipe multiprofissional) e o uso de bases de dados e artigos especializados como forma de atualização profissional. Constatou-se a dificuldade dos profissionais na elaboração de estratégias. O Centro de Documentação também foi apontado como utilizado pelos profissionais, mostrando um panorama diferente da literatura nacional e internacional, além de confirmar a importância do bibliotecário clínico no contexto hospitalar. Tais considerações permitem constatar que, no ambiente informacional da saúde, produtos e serviços precisam ser oferecidos de forma rápida e de acordo com suas necessidades.
\end{abstract}

Palavras-Chave: Comportamento informacional. Análise de domínio. Saúde. Equipe multiprofissional hospitalar. Hospital de Reabilitação de Anomalias Craniofaciais da Universidade de São Paulo - HRAC-USP.

\section{INFORMATIONAL BEHAVIOR OF HEALTH PROFESSIONALS AT THE HOSPITAL OF REHABILITATION OF CRANIOFACIAL ANOMALIES OF THE UNIVERSITY OF SÃO PAULO - HRAC - USP}

\begin{abstract}
Since more than 50 years, information behavior studies seek to describe the process of searching, access, use and retrieval of information. In the area of Information Science, over the years, various studies were performed in order to meet the growth in the number of studies and their contribution to improvements in information media services. The health area is a rich environment of research due to the particularities of its professionals and the diversity of backgrounds where they work together in multiprofessional teams. It is the case of the team attending cracked bones ate the
\end{abstract}


Hospital de Reabilitação de Anomalias Craniofaciais the University of São Paulo (HRAC - USP), in the city of Bauru, considered by World Health Organization as reference in the service to people with cleft lip palate. Looking to describe the search process needs found during rotinary attendance, our goal was to identify the main sources of research used health professionals and the informational needs in attendance. The data collection was mad questionnaires to the professional service team encharged of cleft lip palate at the Local Documentation Center. The results show that query to colleagues within the multiprofessional team is the primary source of research, then articles and specialized sites. The documentation center was also appointed as used by the professionals, showing a different perspective of the national and international literature, and confirming the importance the clinical librarian in the health area in hospitals. Such considerations let us conclude that professionals have little time available for research, so in the health informational environment it is need to ofter products and services of fast performance according to the needs of the professionals, as to the role of multiprofessional teams in hospitals become the great differential in health, because they allow the subject to be seen as a whole facilitating the desired process of humanization in hospital.

Keywords: Information behavior. Health professionals. Multidisciplinary team. Information on health. Craniofacial anomalies.

\section{INTRODUÇÃO}

A preocupação com o comportamento demonstrado por usuários na busca e uso da informação remonta aos anos de 1940 e, até os dias de hoje, ainda é um assunto em descoberta constante e de significância ímpar, uma vez que essa busca se diferencia entre sujeitos e grupos diversos. Dessa forma, estudar o comportamento informacional de determinado grupo é relacioná-lo à necessidade, à busca e ao uso da informação dentro de determinado contexto. Todos os dias, inúmeros novos estudos são publicados em diversos suportes, e a internet tornou-se um repositório gigantesco onde se encontram informações diversas: boas ou ruins, completas ou fragmentadas, com facilidades em termos de acesso, pelo menos parcial, a documentos para os diversos profissionais que precisam se manter atualizados e competitivos no mercado de trabalho, primando por melhores condições de trabalho ou relações profissionais.

Diante disso, surgem as dúvidas: como tais profissionais se atualizam? Que tipos de fontes de pesquisa utilizam? Fazem a própria pesquisa ou solicitam a terceiros? Quando a dúvida surge durante o atendimento, o que fazem? Pensando nesses problemas, algumas áreas dedicam-se ao aprimoramento desse processo de busca pela informação. É o caso da Ciência da Informação, que abriga estudos de comportamento informacional, trabalhando com questões de uso, acesso e recuperação da informação. 
Os estudos de comportamento informacional surgiram há mais de 50 anos (WILSON, 2010) e, durante este tempo, inúmeros trabalhos foram produzidos a respeito desse tema. Assim, este estudo, que segue a abordagem de domínio (HJORLAND, 2002, 2004), investiga o comportamento informacional de profissionais da área de saúde, especificamente os

A escolha do Hospital de Reabilitação de Anomalias Craniofaciais da Universidade de São Paulo para a realização desta pesquisa deu-se em virtude de ser este hospital reconhecido como centro de excelência no diagnóstico e tratamento de anomalias craniofaciais e síndromes relacionadas pela Universidade de São Paulo e como "referência mundial” pela Organização Mundial da Saúde (OMS) (RODELLA, 2017). O Hospital está vinculado à Universidade de São Paulo (USP) e seus atendimentos são prestados exclusivamente pelo Sistema Único de Saúde (SUS). Além disso, ele reúne grandes equipes multidisciplinares de profissionais especializados da área da saúde.

Sendo assim, o objetivo deste artigo é apresentar parte dos resultados do estudo que retratou o comportamento informacional dos membros de uma equipe multidisciplinar composta por profissionais especializados da área da saúde em seu cotidiano, evidenciando suas buscas e acessos à informação. Ao longo do estudo, reafirmou-se a contribuição do bibliotecário na área da saúde e o crescimento dos estudos sobre a temática do comportamento informacional na atualidade.

\section{DESENVOLVIMENTO}

A elaboração do referencial teórico da pesquisa deu-se por meio do levantamento bibliográfico e análise do material encontrado em publicações científicas nacionais e internacionais impressas e eletrônicas nos idiomas português, inglês e espanhol, no período de 2000 a 2014, conforme se verá a seguir.

De acordo com Branco (2006, p. 9), “[...] a área da saúde há algumas décadas vem constituindo um segmento produtor de importante e significativo volume de dados. Essa característica deve-se a crescente complexidade do próprio sistema de saúde" e isso afeta de forma clara o universo e o comportamento informacional.

Comportamento informacional é o comportamento humano relacionado às fontes e canais de informação, seja na busca ativa ou passiva, e ao uso dessa informação. Estão incluídas também a comunicação pessoal, presencial e a recepção passiva da informação 
(WILSON, 2000). Pettigrew, Fidel e Bruce (2001) e Gasque e Costa (2010) complementam o conceito anterior, descrevendo o comportamento informacional como um campo proveniente dos estudos de usuários, constituindo-se uma evolução dele.

Há na literatura diversos modelos sobre comportamento informacional, voltados a diferentes grupos de indivíduos ou contextos. Um modelo pode ser descrito como uma estrutura para pensar sobre determinado problema, podendo evoluir para uma declaração das relações entre proposições teóricas, e procura descrever os passos de uma busca, bem como relações, causas, consequências e estágios, que muitas vezes são representados por diagramas (CASE, 2012).

Para Bates (2006), o modelo é uma tentativa de estruturar as ideias que são utilizadas para descrever etapas de determinado fenômeno. Assim, apenas após essa explanação é que se obtém a teoria propriamente dita. No caso deste estudo, foram tratados os modelos de Wilson (2000), e, embora a área da saúde possua alguns outros localizados ao longo do estudo, não há nenhuma pretensão de se escolher o melhor, buscou-se apenas discutir sobre os já existentes.

Quanto às terminologias, Gasque e Costa (2010, p.21) afirmam que há uma grande diferença entre o comportamento informacional e os estudos de usuários.

Uma diferença crucial está relacionada à mudança conceitual observada, a
qual denota a ampliação da visão epistemológica dos estudos. Tal mudança
refere-se, especialmente, à nova terminologia adotada, que passa de "estudos
de usuários" ou "necessidades e uso de informação" para "comportamento
informacional de usuários". Trata-se, contudo, não somente da alteração
terminológica, mas, sobretudo, de mudança paradigmática, resultado de
transformações no modo como o tópico é definido e abordado e na forma
como é investigado.

Como resultados das mudanças, três novas abordagens surgiram: a cognitiva, que examinava o sujeito a partir de seu conhecimento, convicções e crenças sobre o mundo; a social, baseada em significados e valores atribuídos em diferentes contextos; e a multifacetada, que integra opiniões para a compreensão do comportamento informacional. Assim, ampliou-se a compreensão sobre estudos de comportamento enquanto processos, além de permitir o aperfeiçoamento dos estudos qualitativos e seus métodos múltiplos, proporcionando maior consistência teórica na argumentação e no crescimento de pesquisas em todo o mundo.

Ao longo das décadas, estudos de comportamento informacional - CI (IB Information behavior) têm sido feitos em diferentes contextos profissionais (educação, leis, 
negócios, saúde, etc.), mas seu amadurecimento, segundo Bates (2010), veio com as publicações de Case, em 2002. Em seu livro Looking for information (2012), o autor aborda a complexidade da investigação sobre os estudos de comportamento informacional e as tentativas de definir as informações, descrever necessidades e explicar o uso da informação, tornando-se uma fonte básica do assunto.

Nesse contexto, estudos sobre o comportamento informacional têm crescido em território nacional, nos últimos anos, e as universidades que mais contribuem para esse amadurecimento foram identificadas no estudo realizado por Taga e Blattmann (2012). No entanto, especificamente sobre a saúde, foram encontrados apenas cinco trabalhos cujos conteúdos envolvem a saúde (ANDRADE, 2008; SILVA, 2008; SANTOS, 2009; RODRIGUES, 2009; MACIEL ALBUQUERQUE, 2010), sendo quatro estudos em nível de mestrado e um doutorado, nas seguintes instituições: Universidade Federal de Minas Gerais (UFMG), Universidade Federal da Paraíba (UFPB) e Universidade de Brasília (UNB).

\subsection{O COMPORTAMENTO INFORMACIONAL DOS PROFISSIONAIS DA SAÚDE}

O trabalho de Andrade, da UFMG (2008), abordou a tomada de decisão e os sistemas de informação em saúde, analisando o fluxo informacional do NUPAD (Núcleo de Ações e Pesquisa em Apoio Diagnóstico), responsável pela triagem neonatal no estado de Minas Gerais, na perspectiva dos seus gestores. Por meio de entrevistas, foi determinada a influência dos sistemas de informação na tomada de decisão e seu impacto na formação de políticas e estratégias.

Silva, da UFPB (2008), estudou o modelo de aceitação de tecnologia (TAM) aplicado ao sistema de informação da Biblioteca Virtual de Saúde (BVS) nas escolas de medicina. Por meio de questionário, os alunos de medicina da região metropolitana de Recife responderam sobre a arquitetura informacional da BVS.

Santos, da UNB (2009), realizou uma avaliação sobre a demanda e oferta de informação em saúde, considerando como universo de pesquisa o Banco de Informações técnicas em saúde (BITS) do Disque saúde e a literatura bibliográfica disponível na BVS, ambos do Ministério da Saúde (MS). Como resultado, o estudo mostrou a necessidade de padronização da linguagem utilizada pelo BITS e pelo tesauro do MS. 
Rodrigues (2009), também da UNB, buscou identificar a necessidade de informação dos Conselheiros de Saúde. Utilizou a abordagem do sense-making, de Dervin, e, baseando-se no estudo de Wilson e Martinez-Silveira, propôs o modelo de comportamento informacional dos Conselheiros da Saúde.

Maciel Albuquerque (2010), da UFPB, pesquisou a satisfação de médicos da Unidade de Saúde da Família, no Distrito Sanitário de João Pessoa, por meio de entrevistas e questionários, a fim de identificar as necessidades e o uso da informação. O cotidiano dos médicos nas unidades, segundo o autor, foi evidenciado pelo trinômio preconizado pelo modelo sense-making, de Dervin.

Autores como Johnson et al. (2003; 2006; 2009), Ajayi (2005), Dee e Stanley (2005), Crespo; Caregnato (2006), Davies (2007), Albuquerque; Oliveira e Ramalho (2009), Cullen; Clarkt; Esson (2011), Nwezeh; Shabi; Shabi (2011), Gilman (2011), O’Leary e Ni Mhaolrúnaigh (2011), Hunt, Cimino e Koziol (2013) e Machado e Barbosa (2014) são alguns dos que possuem estudos sobre a questão do acesso, busca e uso da informação, enquanto Camargo Jr (2003), Pereira (2008), Al-Dousari (2009), Savi e Silva (2010), Savi e Silva (2011), Botello-Harbaum et al. (2013) e Galvão (2014a) identificam as fontes de pesquisa mais utilizadas pelos profissionais da saúde, especificamente (WELLICHAN, 2015).

\subsection{CONTEXTUALIZAÇÃO DA PESQUISA: O HOSPITAL DE REABILITAÇÃO DE ANOMALIAS CRANIOFACIAIS DA UNIVERSIDADE DE SÃO PAULO - CENTRINHO}

De acordo com dados institucionais, o Centrinho possui na atualidade 109.049 pacientes matriculados (entre pacientes com fissura labiopalatina e anomalias craniofaciais, além da saúde auditiva e outros programas) e estão em tratamento 63.551 (sendo $63 \%$ do Estado de São Paulo). A equipe é formada por profissionais da medicina, odontologia, fonoaudiologia e outras especialidades da saúde e áreas de apoio, além de docentes da Faculdade de Odontologia da Universidade de São Paulo - FOB-USP que atuam em ambas as instituições (RODELLA, 2017).

O hospital busca a reabilitação e inclusão social das pessoas com fissura labiopalatina, malformação craniofacial congênita e deficiência auditiva. Como alguns tratamentos acontecem em longos períodos, pacientes e familiares se envolvem em diversas atividades que humanizam o atendimento durante o período de internação e proporcionam a interface com a comunidade. 
O tratamento de um portador de fissura, por exemplo, é oferecido desde o nascimento até 20 anos de idade (dependendo da gravidade e comprometimento de cada caso). Além das cirurgias primárias (de lábio aos três meses e de palato com um ano), podem ser necessárias ainda cirurgias secundárias, que buscam a melhora no lábio, nariz, fala, além de correções no nariz (na adolescência), cirurgia de enxerto ósseo alveolar, cirurgias otológicas e ortognósticas, o que acontece mediante a assistência multiprofissional existente no hospital.

Em sua dimensão educativa, desde 1995, o Centrinho oferece cursos de pósgraduação stricto, lato sensu e de extensão, além de cursos de atualização, aprimoramento profissional, prática profissionalizante, residência médica em otorrinolaringologia, residência multiprofissional em síndromes e anomalias craniofaciais e residência em saúde auditiva, além de recentemente ser aprovada a criação do curso de Medicina, num projeto que envolve Estado, município e universidade (USP, 2017).

\section{METODOLOGIA}

A pesquisa, desenvolveu-se por meio de um estudo de caso, cujo local de realização foi o Hospital de Reabilitação de Anomalias Craniofaciais, vinculado à Universidade de São Paulo USP - campus de Bauru, SP.

Para a coleta de dados junto aos profissionais de saúde, optou-se pelo questionário elaborado com base em pesquisas da área, visando atender aos objetivos da pesquisa, com oito questões de múltipla escolha e duas dissertativas, divididas em duas partes: na primeira (perguntas de um a cinco, de múltipla escolha), buscou-se a caracterização do sujeito com questões referentes ao gênero, faixa etária, formação e tempo de atuação dos participantes; na segunda parte, com as duas questões dissertativas e três questões de múltipla escolha, objetivou-se levantar informações sobre a atuação profissional e a necessidade informacional dos profissionais em atendimento.

Visando garantir a fidedignidade, a validade, clareza e acessibilidade dos dados, foi realizado um pré-teste para identificação da necessidade de possíveis ajustes, aprimorando o instrumento selecionado para a coleta de dados com os profissionais. Após a aplicação do 
pré-teste e feitas as alterações necessárias, submeteu-se o projeto à Plataforma Brasil para envio de documentos, informações e cadastramento sobre o estudo.

Como critério para seleção dos sujeitos, definiram-se os profissionais que comporiam a equipe de atendimento aos pacientes com fissura labiopalatina do HRAC - USP. Identificou-se, em janeiro de 2014, que 196 profissionais comporiam a equipe que realizaria os atendimentos no Centrinho. Verificou-se que os maiores contingentes de profissionais se situavam nas carreiras de médicos, cirurgiões dentistas, enfermeiros, fonoaudiólogos, seguidos de assistentes sociais e psicólogos (estes considerados como equipe de apoio). Assim, a coleta de dados foi realizada para esse grupo de 170 profissionais (número referente ao total de profissionais disponíveis na instituição no momento da coleta de dados). As categorias de respondentes da pesquisa foram estabelecidas de acordo com os seis grupos de profissionais: médicos, cirurgiões dentistas, enfermeiros, fonoaudiólogos, assistentes sociais e psicólogos.

Todos os sujeitos da pesquisa foram contatados inicialmente por e-mail para apresentação da pesquisa e convite para participação. Após alguns contatos reenviados sem sucesso, houve o contato pessoal com os profissionais, com a ajuda de duas alunas do doutorado no HRAC - USP, que auxiliaram na abordagem dos profissionais, na entrega do questionário e do Termo de Consentimento Livre e Esclarecido (TCLE).

Enfim, participaram 56 profissionais que atendiam aos casos com fissura labiopalatina, da equipe multidisciplinar, formada por profissionais dos seis grupos envolvidos e representou 32,94\% do total de 170 profissionais, incluindo 7 homens e 49 mulheres, com idade variável entre 31 e 60 anos, com maior participação de psicólogos e os que menos participaram foram os médicos.

Quanto ao nível de formação na pós-graduação dos 56 profissionais participantes da pesquisa, no momento da coleta havia 21 especialistas, 8 mestres e 27 doutores, e o tempo de atuação dos sujeitos no hospital variou entre menos de 5 anos e mais de 11 anos.

As respostas obtidas foram tabuladas e analisadas utilizando-se métodos descritivos, enquanto as dissertativas foram analisadas, interpretadas e agrupadas de acordo com os temas semelhantes e os objetivos propostos inicialmente pelo estudo. 


\section{APRESENTAÇÃO DOS RESULTADOS E DISCUSSÃO}

A consulta à equipe multiprofissional foi a opção mais assinalada entre os profissionais das seis áreas envolvidas, sendo que cinco eram médicos, seis cirurgiões dentistas, oito fonoaudiólogos, cinco assistentes sociais e sete psicólogos. Tal conduta pode estar relacionada às características da clientela atendida e ao modo de atuação do hospital, cuja equipe foi desenvolvendo seus próprios protocolos. O trabalho especializado e interdisciplinar no processo de reabilitação das anomalias craniofaciais requer conhecimento técnico e experiência na aplicação das estratégias, compreensão sobre o contexto no qual o paciente e sua família estão inseridos, bem como sobre suas expectativas. Observa-se que na área da saúde, principalmente entre equipes, a questão do compartilhamento de informações é comum e necessária para o andamento dos atendimentos e decisões de condutas diante dos tratamentos. A consulta a colegas de profissão ou equipes multiprofissionais foi também a fonte de pesquisa mais apontada nos estudos de França (2002), Ajayi (2005), MartinezSilveira (2005), Davies (2007), Al-Dousari (2009), Silva (2010), Cullen, Clark e Esson (2011), Nwezeh, Shabi e Shabi (2011), Giilman (2011), Sigolo (2012), conforme citado por Wellichan (2015).

Cinco profissionais citaram outras fontes como escolha, sendo três enfermeiros, um fonoaudiólogo e um psicólogo. Entre as fontes indicadas estão: três ocorrências de consulta em sites especializados, ao buscador Google, livros específicos e consulta ao intersetores (a folha no início do prontuário, em que os profissionais fazem as anotações de interesse de outras áreas. Também funciona como um "encaminhamento" quando solicitam avaliação de outro profissional. Dois profissionais (um da psicologia e um do serviço social), que apontaram o prontuário como uma fonte de pesquisa, também fazem uso dos intersetores para melhor entendimento durante a consulta, e quatro profissionais não identificaram nenhuma fonte de preferência; uma ocorrência de profissional que afirmou não ter dúvida; e uma ocorrência que apontou como fonte a leitura de artigos especializados e a confirmação de dúvidas com médicos plantonistas do dia, quando necessário.

De acordo com os resultados apresentados, a fonte mais utilizada corresponde aos profissionais da área da saúde, seguidos de profissionais da área médica, artigos científicos em base de dados, periódicos especializados, sites especializados, biblioteca do HRAC - 
USP, coleção particular, protocolos e guidelines, buscadores na internet e relatórios de pesquisa.

França (2002), que também estudou o comportamento informacional de profissionais da saúde, focado em médicos e enfermeiros do Programa de Saúde da Família, já apontava preferências similares a este estudo. Visitas domiciliares e reuniões com grupos-controle (especificidades do programa citado), além da comunicação com colegas de profissão, foram as fontes mais citadas, seguidas dos prontuários, exames laboratoriais, coleções pessoais, livros, periódicos científicos, boletins informativos e colegas especialistas da própria instituição.

A biblioteca do HRAC - USP apareceu com 22 ocorrências, sendo apontada por todas as categorias envolvidas na coleta de dados, sendo mais utilizada por enfermeiros, médicos e cirurgiões dentistas e menos utilizada por fonoaudiólogos e assistentes sociais. As Seções de Documentação e Informação, Apoio à Pesquisa e Referência Especializada em Malformações Congênitas Craniofaciais atuam juntas e constituem o Centro de Documentação Cientifica e Informação do HRAC - USP, cuja missão é apoiar as atividades clínicas de ensino, pesquisa e extensão, oferecendo suporte informacional necessário para um sistema modelo de prestação de serviços, proporcionando um atendimento diferenciado e especializado graças à diversificação de documentos que compõem o acervo e que contribui para o acompanhamento evolutivo dos pacientes e para a consulta dos profissionais, quando necessário.

Dentre as bases de dados, nota-se que a PubMed foi preferência geral entre as categorias, sendo também a preferida entre psicólogos e médicos. No estudo de Sigolo (2012), a base de dados PubMed também foi a mais citada por médicos e cirurgiões dentistas, representando mais uma similaridade com a literatura nos estudos da área de comportamento informacional. A MedLine, Scielo e LILACS também foram citadas pelos profissionais. Na categoria "outras", foram citadas: SIBI (Sistema Integrado de Bibliotecas da USP), DEDALUS (Banco de Dados Bibliográficos da USP), Cleft Palate Journal (Jornal internacional com publicações especializadas na área de anomalias craniofaciais da ACPA American Cleft Palate-Craniofacial Association), Bireme (Biblioteca Virtual da Saúde) e OMIN (Base de dados de genes e desordens genéticas). Fontes de pesquisa específicas, tais como Dysphagia, Hera, Speech e Chew, cujos conteúdos estão relacionados à deglutição e distúrbios apresentados por indivíduos com fissura labiopalatina ou anomalias craniofaciais, também foram citadas. Entre os buscadores, o Google foi indicado por enfermeiros, e 
psicólogos. Já o Google Scholar foi citado por médicos, cirurgiões dentistas, enfermeiros, fonoaudiólogos e pelos psicólogos.

Relacionando, especificamente, a questão da fonte de informação à internet, os profissionais foram questionados sobre as dificuldades encontradas ao buscar as informações, e a maior dificuldade apontada foi selecionar o que de fato é relevante diante dos resultados recuperados em suas buscas. A segunda categoria mais pontuada foi a falta de tempo para pesquisar. Em terceiro surgiu a dificuldade na definição de palavras-chave para a busca. Para Cardoso (2004, p.5), definir a necessidade de informação em termos de busca é uma tarefa difícil, "[...] pois existe uma distância semântica entre a real necessidade dos usuários e o que ele expressa na consulta formulada". Sendo assim, a dificuldade em definir as palavras-chave para as buscas pode estar relacionada à dificuldade de expressar as necessidades reais informacionais e torná-las seus termos de busca (SIGOLO, 2012).

\section{CONSIDERAÇÕES FINAIS}

A questão da falta de tempo no comportamento informacional de profissionais da saúde é um fator de grande importância, não só pelo fato de interferir no processo de busca, uma vez que as longas jornadas dificultam a dedicação para pesquisas e atualizações exigidas pela sociedade em forma de tratamentos e novas descobertas na área, mas também por envolver a questão de recursos disponíveis no ambiente de trabalho e habilidades para realizar os processos de busca pela informação. Mas se o tempo é fator de urgência na área da saúde, o profissional no hospital precisa de informação em tempo hábil, necessitando de pessoal qualificado e preparado para oferecê-la. Seja durante a formação na universidade ou na formação continuada, o bibliotecário clínico precisa estar apto para atender à demanda de sua realidade.

A equipe multiprofissional do HRAC - USP não se restringe ao grupo que atende aos casos de fissura labiopalatina. O hospital oferece serviços em saúde auditiva, conta com uma demanda de igual tamanho e importância e atende aos complexos casos de síndromes associadas às anomalias craniofaciais. Portanto, ainda há especificidades e necessidades diferentes entre as equipes do hospital e, por isso, há muito a ser feito, tanto em termos de 
apoio aos atendimentos das equipes quanto em relação às pesquisas, sem falar nos serviços de auxílio à organização e guarda de documentação clínica.

Com relação ao comportamento informacional dos profissionais, foi possível verificar, a partir dos resultados colhidos, que eles realizam pesquisas com frequência, o que configura a predominância da busca ativa por informação.

Notou-se a preferência por fontes de informação informais (consultas a colegas e/ou à equipe multiprofissional), bem como o uso de bases de dados e artigos especializados como forma de atualização profissional. Constatou-se a dificuldade dos profissionais na elaboração de estratégias e na definição de palavras-chave para a busca informacional, o que acaba por influenciar os resultados das pesquisas e mostra a necessidade não só de mais treinamentos específicos, mas, também, de o bibliotecário clínico estar atuando junto às equipes, auxiliando nos processos de busca e recuperação da informação, conforme apontado por autores citados neste trabalho.

Assim, acredita-se que a análise realizada neste trabalho não esgota as discussões sobre o comportamento informacional do grupo pesquisado, mas pode contribuir com subsídios para estudos futuros.

\section{AGRADECIMENTOS}

Agradecemos aos profissionais do Hospital de Reabilitação e Anomalias Craniofaciais da Universidade de São Paulo - HRAC - USP pela participação e colaboração. 


\section{REFERÊNCIAS}

ANDRADE, André Queiroz de. A tomada de decisão e sistemas de informação em saúde. 2008. 118 f. Dissertação (Mestrado) - Escola de Ciências da Informação, Universidade Federal de Minas Gerais. Disponível em: http://www.bibliotecadigital.ufmg.br/dspace/bitstr eam/1843/ECIC-7XMFGC/1/dissertacao_andre_queiroz.pdf. Acesso em: 12 maio 2012.

BATES, M.J. Information behavior. Encyclopedia of Library and Information Sciences, 3rd Ed. 2010. Disponível em: http://pages.gseis.ucla.edu/faculty/bates/articles/informationbehavior.html. Acesso em: 25 out. 2013.

BATES, M. J. Fundamental Forms of Information. Journal of the American Society for Information Science and Technology, v. 57, n. 8, p. 1033-1045, 2006.

BRANCO, M.A.F. Informação e saúde: uma ciência e suas políticas em uma nova era. Rio de Janeiro: Fiocruz, 2006.

CARDOSO, O.N.P. Recuperação de informação. INFOCOMP: Journal of Computer Science, 2004. Disponível em: www.dcc.ufla.br/infocomp/artigos/v2/art07.pdf. Acesso em: 8 nov. 2014.

CASE, D.O. Looking for information: a survey of research on information seeking, needs and behavior. 3. ed. London: Academic Press, 2012.

FRANÇA, L.D. O comportamento informacional dos profissionais médicos e enfermeiros do Programa de Saúde da Familia (PSF) - Sistema Único de Saúde. 2002. 160f. Dissertação (Ciência da Informação), UFMG, Belo Horizonte, MG.

GASQUE, K.C.G.D.; COSTA, S.M.S. Evolução teórico-metodológica dos estudos de comportamento informacional de usuários. Ciência da Informação, v. 39, n. 1, p. 21-32, jan./abr. 2010.

HJÖRLAND, B. Fundamentals of knowledge organization. Knowledge organization, v. 30, n. 2, p. 87-111, 2003.

MACIEL ALBUQUERQUE, E. Necessidades e uso de informação: um estudo com médicos de Unidades de Saúde da Família. 2010. 160 f. Dissertação (Mestrado) - Departamento de Ciência da Informação, Universidade Federal da Paraíba. Disponível em: http://dci2.ccsa.ufpb.br:8080/jspui/bitstream/123456789/630/1/NECESSIDADES\%20E\%20U SO\%20DE\%20INFORMA\%C3\%87\%C3\%830\%20\%20um\%20estudo\%20com\%20m\%C3\%A9dicos\%20de\%20Unidades\%20de \%20Sa\%C3\%B Ade\%20da\%20Fam\%C3\%ADlia.pdf. Acesso em: 12 maio 2012.

MARTINEZ-SILVEIRA, M.; ODDONE, N. Necessidades e comportamento informacional: conceituação e modelos. Ciência da Informação, v. 36, n. 1, p. 118-127, maio/ago. 2007.

MARTINEZ-SILVEIRA, M.S. A informação cientifica na pratica médica: estudo do comportamento do médico residente. 2005. 184f. (Dissertação). Disponível em: 
http://repositorio.ufba.br/ri/bitstream/ri/8175/1/DISSERTACAO\%20PRONTA\%2 01.pdf Acesso em: 20 out. 2013.

OSHEROFF, J. A. et al. Physicians' information needs: analysis of questions posed during clinical teaching. Annals of Internal Medicine, v. 114, n. 7, p. 576-581, 1991.

PETTIGREW, K.E.; FIDEL, R.; BRUCE, H. Conceptual frameworks in information behavior. Arist, v.35, 2001. Disponível em: http://projects.ischool.washington.edu/chii/ publications/fidel/conceptualframeworks.pdf. Acesso em: 10 set. 2013.

RODELlA, T. HRAC/Centrinho - USP: 50 anos transformando vidas. Notícias Universidade de São Paulo, Campus Bauru. 22/06/2017. Disponível em: http://www.bauru.usp.br/?p=6205. Acesso em: 10 jul. 2017.

RODRIGUES, C Z. A necessidade de informação dos conselheiros de saúde. 2009. 192 f. Tese (Doutorado) - Faculdade de Ciência da Informação, Universidade de Brasília. Disponível em: http://www.cid.unb.br/m001/M0011000.asp?txtID_PRINCIPAL=2. Acesso em: 12 maio 2012.

SANTOS, Eliane Pereira dos. Estudo sobre demanda e oferta de informação em saúde. 2009. 207f. Dissertação (Mestrado) - Faculdade de Ciência da Informação, Universidade de Brasília. Disponível em: http://bdtd.bce.unb.br/tedesimplificado/tde_busca/arquivo.php?cod Arquivo=5309. Acesso em: 12 set. 2014.

SANTOS, E. P. Estudo sobre demanda e oferta de informação em saúde. 2009. 207f. Dissertação (Mestrado) - Faculdade de Ciência da Informação, Universidade de Brasília. Disponível em: http://bdtd.bce.unb.br/tedesimplificado/tde_busca/arquivo.php?codArquivo $=5309$. Acesso em: 12 set. 2014.

SIGOLO, B.O. Comportamento informacional de cirurgiões dentistas: um estudo junto a ortodontistas da cidade de São Paulo. 2012. 132f. Dissertação (Ciência da Informação), UNESP, Marília, SP.

SILVA, P. M. Modelo de aceitação de tecnologia (TAM) aplicado ao sistema de informação da biblioteca virtual em saúde (BVS) nas escolas de medicina da região metropolitana do Recife. 154 f. 2008. Dissertação (Mestrado) - Departamento de Ciência da Informação, Universidade Federal da Paraíba. Disponível em: http://dci2.ccsa.ufpb.br:8080/js pui/bitstream/123456789/40/1/Disserta\%C3\%A7\%C3\%A30\%20Patr\%C3\%ADcia\%20Silva. pdf. Acesso em: 12 de nov. de 2014.

TAGA, V.; BLATTMANN, U. Comportamento informacional em teses e dissertações na ciência da informação no Brasil entre 2007-2012: revisão de literatura. Biblios, n. 47, p. 3049, 2012.

USP aprova curso de Medicina em Bauru. Jornal da USP, 04 jul. 2017. Disponível em: http://jornal.usp.br/universidade/usp-aprova-curso-de-medicina-em-bauru/. Acesso em: 10 jul. 2017. 
WELLICHAN, D.S.P. Comportamento informacional de profissionais no domínio da saúde: um estudo junto ao Hospital de Reabilitação de Anomalias Craniofaciais da Universidade de São Paulo. 128 f. Dissertação (Mestrado) - Faculdade de Filosofia e Ciências, Universidade Estadual Paulista, Marília, 2015.

WILSON, T.D. Fifty years of information behavior research. ASIST\&T. Annual Meeting Coverage, Bulletin, special section, v. 36, n. 3, p. 27-34, feb/mar 2010.

WILSON, T. D. Human information behavior. Informing Science, v. 3, n. 2, p. 49-55, 2000. Special issue on Information Science Research. Disponível em: http://inform.nu/Articles/Vol3/v3n2p49-56.pdf. Acesso em: 9 nov. 2013. 